\title{
Gerontology
}

Gerontology 2014;60:108-113

DOI: $10.1159 / 000355119$
Received: June 4, 2013

Accepted: August 19, 2013

Published online: November 1, 2013

\section{Walking while Talking and Falls in Aging}

\author{
Emmeline I. Ayers ${ }^{\mathrm{a}}$ Amanda C. Tow ${ }^{\mathrm{a}}$ Roee Holtzer ${ }^{\mathrm{b}}$ Joe Verghese ${ }^{\mathrm{a}}$ \\ ${ }^{a}$ Departments of Neurology and Medicine, Albert Einstein College of Medicine, and ${ }^{b}$ Ferkauf School of Psychology, \\ Yeshiva University, New York, N.Y., USA
}

\section{Key Words}

Falls · Walking · Talking · Divided attention - Older adults . Epidemiology $\cdot$ Cognition · Cohort

\begin{abstract}
Background: While divided attention tasks are recognized as predictors of falls in older adults, a comprehensive examination of this association is lacking. Objective: We examined the validity of a 'walking while talking' (WWT) task for predicting falls. Methods: We studied the associations of 8 selected gait markers measured during WWT (individually as well as domains derived by factor analysis) with incident falls in 646 adults (mean age 79.9 years; $61 \%$ women) enrolled in an aging study who received quantitative gait assessments. Cox regressions adjusted for multiple potential confounders and normal-pace walking were used to examine the associations. Results: Over a mean follow-up of 2.6 years, 337 participants (52\%) fell. Step length was the only individual WWT parameter that predicted falls [hazard ratio (HR) 0.98; $p=$ 0.034]. Factor analysis identified 3 gait domains, of which only the pace factor predicted falls (HR 1.31; $p=0.002$ ). Results remained robust after adjusting for multiple potential confounders and accounting for normal-pace walking. Conclusions: WWT performance was a significant predictor of falls. Gait domains in WWT should be further studied to improve current fall risk assessments and to develop new interventions.

(c) 2013 S. Karger AG, Basel
\end{abstract}

\section{Introduction}

Lundin-Olsson et al. [1] observed that nursing home residents who stopped walking when talking had a higher risk of falls. This seminal observation has since spurred exploration of dual-task methods such as the 'walking while talking' (WWT) test to understand fall risk by studying individuals as they walk while simultaneously conducting a cognitively demanding task. The WWT paradigm affords the opportunity to manipulate attention demands and measure the effect of taxing the attention system on gait performance. The decrement in gait performance during WWT is considered a measure of dual-task cost that arises from the two tasks interfering with each other and competing for the same brain resources $[2,3]$.

A systematic review by Beauchet et al. [4] concluded that dual tasks were strong predictors of falls. However, 4 of the 15 studies reviewed had negative results [4]. Methodological limitations of previous studies identified in this review included small samples, limited follow-up and lack of standardization for dual-task procedures $[4,5]$. Moreover, most studies only report changes in gait velocity, though other gait variables might predict falls. For instance, we reported that stride length variability during normal-pace walking was a stronger predictor of falls

E.I.A. and A.C.T. contributed equally as first authors.

\section{KARGER}

E-Mail karger@karger.com

www.karger.com/ger
(C) 2013 S. Karger AG, Basel

0304-324X/13/0602-0108\$38.00/0
Joe Verghese, MBBS

Division of Cognitive and Motor Aging, Department of Neurology

Albert Einstein College of Medicine

1165 Morris Park Avenue, Room 301, Bronx, NY 10461 (USA)

E-Mail joe.verghese@ einstein.yu.edu 
than velocity [6]. Beauchet et al. [4] stressed the need for well-designed, prospective population-based studies with large sample sizes to improve the predictive validity of current dual-task-based fall assessment tests.

To address some of the limitations of previous dualtask-based fall assessment studies, we examined the validity of a WWT task for predicting falls in 646 communitydwelling older adults. Establishing the role of WWT in falls has potential to improve risk assessments and provide mechanistic insights to guide development of new fall interventions.

\section{Methods}

\section{Study Population}

The Einstein Aging Study (EAS) is a prospective cohort study of community-dwelling residents of Bronx County. The primary aim of the EAS was to identify risk factors for dementia [7]. The study design has been reported previously $[8,9]$. Potential participants (age 70+ years) identified from population lists of Bronx County were contacted first by letter, then telephone, to explain the purpose and nature of the study. Telephone interviews included verbal consent, medical history and cognitive screens. Exclusion criteria included severe auditory or visual loss, inability to ambulate and institutionalization. Participants returned annually for clinical, cognitive and mobility assessments and were contacted by telephone every $2-3$ months to assess function and falls. Informed consent was obtained at enrollment according to protocols approved by the Einstein Institutional Review Board.

\section{Gait Assessment}

Quantitative gait studies were conducted at baseline using a computerized walkway $(180 \times 35.5 \times 0.25$ inches $)$ with embedded pressure sensors (GAITRite, CIR Systems, Pa., USA) in a quiet well-lit hallway. Participants walked on the mat at their normal pace while computer software recorded gait variables as the mean of 2 trials. To account for initial acceleration and terminal deceleration, data collection started and stopped 3 feet from either end of the walkway edge. The GAITRite system is widely used and has excellent reliability $[5,6,10]$.

\section{Walking while Talking}

Subjects walked on the mat, as described above, while reciting alternate letters of the alphabet. They were instructed to pay equal attention to walking and talking [3]. To reduce learning effects, subjects were randomly assigned to start with the letter ' $\mathrm{A}$ ' or ' $\mathrm{B}$ '. The number of errors and correctly recited alternate letters were recorded. Testers intervened only if subject safety was an issue. Based on our and other locomotion and falls studies $[6,11,12]$, we selected the following 8 gait variables: velocity (centimeters per second), cadence (steps per minute), step length (centimeters), swing (percent), stance (percent), double support (percent), step time variability (standard deviation; SD) and swing time variability (SD).

We have reported high reliability of our current WWT protocol [3] and its association with cognitive processes $[13,14]$ relevant to dual-tasking. In a preliminary study of WWT, using a previous version of the protocol without quantitative gait assessments, we reported that velocity during WWT predicted falls [9].

\section{Falls}

Falls were defined as unintentionally coming down to the floor or a lower level not due to a major intrinsic or extrinsic event [15]. Participants were interviewed about occurrence of falls at baseline and by telephone every 2-3 months [6]. They were also asked about falls in the prior year during annual clinic visits. Consistency between participant report of falls on the phone and in-person interviews was reported to be highly reliable in our previous study; all participants who reported a fall at 6 months on the phone recalled the fall at the subsequent in-person interview [9].

\section{Covariates}

The presence or absence of depression, diabetes, heart failure, hypertension, angina, myocardial infarction, strokes, Parkinson's disease, chronic obstructive lung disease and arthritis was used to calculate a summary illness index [6]. Activities of daily living were assessed using a disability scale for community-based cohorts [16]. General cognitive status was assessed by the Blessed InformationMemory-Concentration test [17]. Depression was assessed using the 15-item Geriatric Depression Scale (GDS) [18]. Visual acuity was measured with Snellen's chart $(<1 / 200=$ low acuity). Clinical gait abnormalities were diagnosed by visual inspection of walking patterns by study clinicians and have been reported to predict falls in our cohort $[8,19]$. Balance and lower extremity strength was assessed by the time taken to rise from a chair 5 times unassisted [20] Unipedal stance time was recorded as the time balanced on one foot without support [21].

Statistical Analysis

Baseline characteristics of fallers and nonfallers were compared with descriptive statistics. Cox proportional hazards models were used to compute hazard ratios (HRs) with $95 \%$ confidence intervals to predict incident falls based on baseline WWT performance on the 8 selected parameters. Quantitative gait variables are highly correlated, and independent effects are difficult to discern. Hence, the principal component method was used to conduct an orthogonal varimax rotated factor analysis of the 8 quantitative gait variables to derive statistically independent gait domains $[6,11,12$, 22]. The time scale was follow-up time (days) to an incident fall or final contact. All models were adjusted for age, sex and education. Further adjustments included the illness index, prescription medication count, Blessed score, GDS score, chair rise time, falls in the year prior to enrollment and presence of gait abnormalities. Variables included in the models were identified as fall risk markers in bivariate analysis and in our prior study [6].

We reported that quantitative gait variables during the normalpace walking condition predict falls [6]. Hence, to examine the incremental validity of WWT for falls we conducted sensitivity analyses accounting for normal walking velocity and clinical gait status in the fully adjusted model. Additionally, we adjusted for normal walking stride length variability, as it has previously been reported to be the best predictor of falls [6].

Proportional hazards assumptions of all models were tested graphically and analytically and were adequately met. All analyses were conducted using SPSS version 20 (SPSS Inc., Chicago, Ill., USA). 
Table 1. Comparison of baseline variables in subjects with and without falls

\begin{tabular}{lccc}
\hline Variable & $\begin{array}{l}\text { Nonfallers } \\
(\mathrm{n}=309)\end{array}$ & $\begin{array}{l}\text { Fallers } \\
(\mathrm{n}=337)\end{array}$ & $\begin{array}{l}\mathrm{p} \\
\text { value }\end{array}$ \\
\hline Age, years & $79.2 \pm 5.5$ & $80.5 \pm 5.4$ & $0.002^{*}$ \\
Female, $\mathrm{n}$ & $176(57)$ & $219(65)$ & 0.054 \\
Education, years & $14.1 \pm 3.1$ & $14.4 \pm 3.2$ & 0.282 \\
Illness index (range 0-10) & $1.17 \pm 1.1$ & $1.16 \pm 0.95$ & 0.980 \\
Medication, n & $3.3 \pm 2.8$ & $3.3 \pm 2.6$ & $0.536^{1}$ \\
Fall in past year, $\mathrm{n}$ & $40(13)$ & $138(40)$ & $<0.001^{*}$ \\
Disability score (range 0-14) & $0.81 \pm 1.3$ & $0.85 \pm 1.2$ & $0.333^{1}$ \\
GDS score (range 0-15) & $2.1 \pm 2.3$ & $2.2 \pm 2.1$ & $0.176^{1}$ \\
Blessed score (range 0-32) & $1.7 \pm 1.6$ & $1.7 \pm 1.5$ & $0.860^{1}$ \\
Visual acuity <1/200, $\mathrm{n}$ & $50(18)$ & $69(21)$ & 0.378 \\
Clinical gait abnormality, $\mathrm{n}$ & $118(39)$ & $131(39)$ & 0.947 \\
Repeat chair rise test, s & $13.8 \pm 4.8$ & $13.7 \pm 4.4$ & 0.838 \\
Unipedal stance, s & $8.7 \pm 8.8$ & $7.9 \pm 7.8$ & $0.406^{1}$ \\
Normal walking velocity, cm/s & $95.8 \pm 22.6$ & $94.3 \pm 22.7$ & 0.382 \\
\hline
\end{tabular}

Values are means \pm SD or numbers (percentage). ${ }^{*}$ Significant $\mathrm{p}$ values.

${ }^{1}$ Calculated by means of the Mann-Whitney U test.

Table 2. Individual WWT gait variables and fall risk

\begin{tabular}{lll}
\hline WWT gait variable & HR & p value \\
\hline Velocity, cm/s & $0.997(0.99-1.00)$ & 0.162 \\
Cadence, steps/min & $0.999(0.99-1.00)$ & 0.711 \\
Step length, cm & $0.989(0.98-0.99)$ & 0.034 \\
Swing, \% & $1.003(0.98-1.03)$ & 0.763 \\
Stance, \% & $0.997(0.98-1.02)$ & 0.776 \\
Double support, \% & $0.994(0.98-1.01)$ & 0.344 \\
Step time SD, s & $0.879(0.57-1.35)$ & 0.554 \\
Swing time SD, s & $1.049(0.60-1.83)$ & 0.867 \\
\hline
\end{tabular}

Values in parentheses represent $95 \%$ confidence intervals. HR values were adjusted for gender, age and education.

Table 3. Factor analysis of $8 \mathrm{WWT}$ gait variables

\begin{tabular}{lrrr}
\hline Gait variable & $\begin{array}{l}\text { Rhythm } \\
\text { factor }\end{array}$ & $\begin{array}{l}\text { Variability } \\
\text { factor }\end{array}$ & $\begin{array}{l}\text { Pace } \\
\text { factor }\end{array}$ \\
\hline Swing, \% & $\mathbf{0 . 9 6 7}$ & -0.020 & 0.195 \\
Double support, \% & $\mathbf{- 0 . 8 0 0}$ & 0.182 & -0.328 \\
Stance, \% & $\mathbf{- 0 . 9 6 7}$ & 0.020 & -0.194 \\
Swing time SD, s & 0.131 & $\mathbf{0 . 9 1 3}$ & -0.078 \\
Step time SD, s & -0.131 & $\mathbf{0 . 9 2 5}$ & -0.023 \\
Cadence, steps/min & 0.248 & $\mathbf{- 0 . 7 1 4}$ & 0.334 \\
Velocity, cm/s & 0.312 & -0.516 & $\mathbf{- 0 . 7 4 2}$ \\
Step length, cm & 0.306 & -0.029 & $\mathbf{- 0 . 8 8 8}$ \\
Variance explained, \% & 34.90 & 31.25 & 20.58 \\
\hline
\end{tabular}

The highest loading variables are shown in bold.

\section{Results}

\section{Study Population}

This study began in September 2004, when we started systematically ascertaining falls in our cohort, and ended in November 2012. Of the 972 EAS participants seen during this period, 131 had no fall assessments, 141 had no WWT assessments (tester or equipment unavailability, refusal or inability to ambulate) and 8 had incomplete WWT. Of the 692 participants with baseline WWT and fall follow-up, we excluded 46 with significant cognitive impairment (Blessed score $>6$ ) to minimize recall bias [23]. The 646 participants completed one or more followup telephone interviews ( $100 \%$ compliance). Over a mean study follow-up period of 638.3 days (range 17-2,604), 337 participants (52\%) reported an incident fall. Mean time to a fall was 479.4 days (range 17-2,192).

The 337 subjects who fell were older $(\mathrm{p}=0.002)$ and had a higher baseline prevalence of previous falls $(\mathrm{p}<$ 0.001) compared to the 309 nonfallers (table 1). Fallers and nonfallers did not differ significantly with regard to other descriptive variables.

\section{Individual Gait Variables}

Step length was the only WWT parameter that predicted falls (table 2), with fallers having significantly shorter steps than nonfallers (mean difference $1.89 \mathrm{~cm}$; $\mathrm{p}=0.042$ ). The number of letters recited (HR 1.010; $\mathrm{p}=$ $0.318)$ and errors during WWT (HR 1.003; $\mathrm{p}=0.879) \mathrm{did}$ not predict falls.

\section{WWT Gait Domains}

Factor analysis of $8 \mathrm{WWT}$ gait variables resulted in 3 independent factors accounting for $86.7 \%$ of variance (table 3). The first factor, denoted 'rhythm', loaded heavily in swing (percent), double support (percent) and stance (percent) and accounted for the largest variance (34.9\%). The 'variability' factor accounted for $31.3 \%$ of variance with swing time SD, with step time SD loading highest. The 'pace' factor accounted for $20.6 \%$ of variance and loaded highest in velocity and step length. The factors were similar to those reported for normal-pace walking by us and other investigators $[6,11]$.

'Pace' was the only factor that predicted incident falls in both models (table 4). For each 1 SD decrement in the 'pace' factor, there was a 31\% increased hazard of falling in model 2 (HR 1.312; $\mathrm{p}=0.002)$. Exclusion of participants with a diagnosis of Parkinson's disease $(\mathrm{n}=3)$ and depression (GDS $>10 ; \mathrm{n}=5$ ) did not materially change the association of the 'pace' factor with falls (HR 1.30; $\mathrm{p}=0.002$ ). 


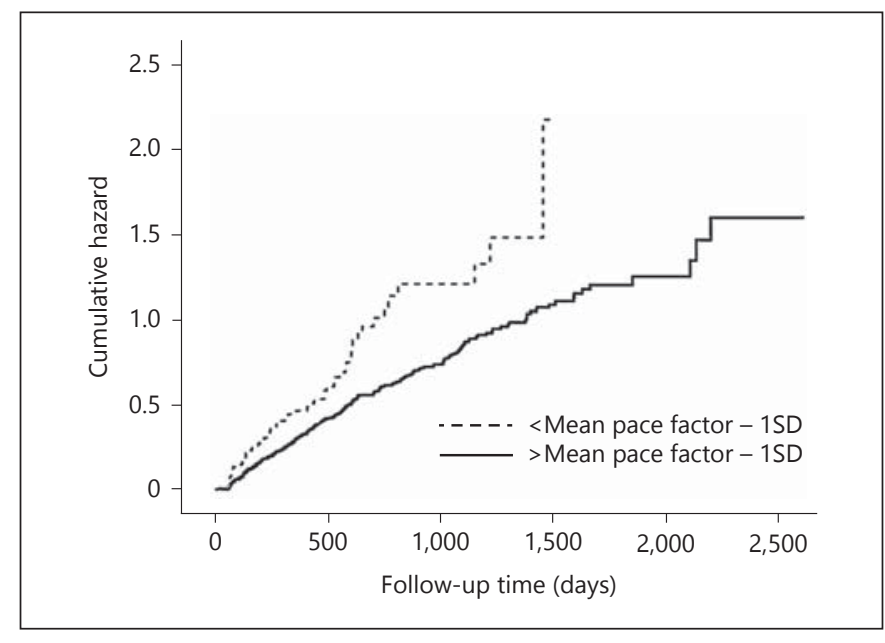

Fig. 1. Kaplan-Meier plots of falls.

Table 4. WWT gait domains and fall risk

\begin{tabular}{llllll}
\hline Factor & Model 1 & & & Model 2 & \\
\cline { 2 - 3 } \cline { 5 - 6 } & HR & p value & HR & p value \\
\hline Rhythm & $0.917(0.81-1.04)$ & 0.161 & & $0.960(0.84-1.09)$ & 0.540 \\
Variability & $1.041(0.92-1.18)$ & 0.539 & & $1.044(0.91-1.19)$ & 0.537 \\
Pace & $1.174(1.04-1.33)$ & 0.011 & & $1.312(1.11-1.55)$ & 0.002 \\
\hline
\end{tabular}

Values in parentheses represent $95 \%$ confidence intervals. Model 1 was adjusted for gender, age and education. Model 2 was adjusted for gender, age, education, illness index, prescription medicines, GDS, Blessed score, chair rise test, clinical gait abnormalities, falls in last year, normal velocity and normal stride length variability.

Kaplan-Meier curves for the risk of falls by WWT 'pace' factor scores are presented in figure 1. We dichotomized the 'pace' factor to compare the fall risk of the slowest $14 \%$ with the rest of the sample. The category with poorer gait performance in the WWT 'pace' domain shows a higher risk of falls. In addition, we examined the fall risk of the fastest $14 \%$ in the WWT 'pace' domain. Results indicated significantly less fall risk (HR 0.685, 95\% confidence interval 0.489-0.960; $\mathrm{p}=$ 0.028 ) for this group in comparison to the rest of the sample.

\section{Influence of Normal-Pace Walking}

Dual-task costs, measured as change in gait velocity between dual-task and single-task conditions, did not predict falls (HR 1.00; $\mathrm{p}=0.412$ ). The 'pace' factor re- mained a significant predictor of falls even after adjusting for normal walking velocity $(\mathrm{HR} 1.297 ; \mathrm{p}=0.002)$ and stride length variability (HR 1.312; $\mathrm{p}=0.002)$.

\section{Discussion}

We investigated the association between WWT and falls in a prospective cohort of 646 community-dwelling older adults. To obtain a comprehensive picture of the complex relationship between WWT and falls we report both individual gait measures and gait domains. In contrast to previous studies, we identified step length, but not velocity or other variables, as the sole gait marker to predict falls in our community-residing elderly cohort. Our results also indicate that the WWT 'pace' factor significantly predicted falls and remained a robust predictor after adjusting for multiple potential confounders [24] as well as baseline normal walking velocity and stride length variability. Step length was the only independent gait variable that predicted falls; however, the 'pace' domain, which primarily consisted of velocity and step length, strengthened the effect of the association. Moreover, supplemental analyses comparing groups in the fastest and slowest $14 \%$ of the WWT 'pace' domain to the rest of the sample illustrated additional support for the predictive validity of slow gait, derived from factor analysis, for predicting falls in this cohort.

To our knowledge, this is the largest study of the predictive validity of WWT as a predictor of falls that has been conducted to date in a prospective cohort. Moreover, as recommended in a recent review article by Beauchet et al. [4], assessments were conducted using a standardized and previously validated dual-task test [9], and results accounted for multiple confounding variables including cognition and health status as well as singletask walking variables to improve the predictive validity of WWT [4].

Unlike our study, most previous dual-task and falls studies were conducted in smaller or select samples, had limited follow-up, lacked standardized protocols or did not account for single-task walking [4]. Aside from a study by Kressig et al. [5] which reported that greater stride time variability measured during a dual task increased the fall risk for elderly inpatients, exploration of dual-task gait variables other than speed to predict falls is limited. Velocity was only significant in our study as a fall predictor as a contributor to the 'pace' domain, supporting the incremental contribution of the factor analysis approach. Our study also indicated that dual-task costs, 
measured by gait slowing between WWT and normalpace walking, did not predict falls. These methodological differences may help explain discrepant results in some studies that reported gait slowing as the only measure of WWT [4]. Two recent publications by Lord et al. [11] and Hollman et al. [12] that were influenced by our novel application of factor analysis to studying gait $[6,13]$ support the utility of this statistical approach to capture the multidimensional characteristics of gait. All variables included in the factor analysis contribute to the final factors that are derived, but the magnitude of their contribution to the different factors will vary. Identifying domains of gait through the factor analysis is a more powerful and sensitive approach for understanding potential underlying motor, cognitive or behavioral disturbances. Hence, it is appropriate to study the factors separately from the individual variables.

Our findings in the current and previous studies of the same cohort $[6,9]$ highlight differences between single- and dual-task conditions with respect to the predictive validity of individual gait variables and domains for falls. For example, step length was the only individual WWT gait variable that predicted falls, while velocity, stride length, double support percent, stride length variability and swing time variability during normal walking were reported to predict falls in the same cohort [6]. The 'pace' factor accounts for the greatest variance in normal-pace walking $[11,22]$ but the least in the dual-task condition. Studies of single-task normal walking in the same cohort [6] showed that 'variability' and 'rhythm' factors rather than 'pace' predicted falls. Moreover, after accounting for baseline normal walking velocity and stride length variability, 'pace' remained a robust predictor of falls.

The ability to recite alternate letters of the alphabet while walking has been shown to involve similar cortical regions as gait [25]. Specifically, attention, executive function and memory regions of the prefrontal cortex are involved in completing both tasks [22]; therefore, the dual-task paradigm could reveal early indicators of dysfunctions in particular brain regions $[25,26]$. Different brain processes and substrates have been correlated with WWT-like tasks and normal walking [22, 27-29]. Doi et al. [27] found an association between dual-task costs in trunk instability and brain atrophy, suggesting trunk instability as a marker of brain volume decline. Change in gait speed between single and dual tasks was greater in adults with mild cognitive impairment with decreased cortical volume of the primary motor cortex [28]. Cognitively impaired adults with greater subcortical hyperin- tensity severity have also been shown to perform worse in dual tasks compared to normal controls [29]. These findings suggest that dual- and single-task walking assessments may tap into different fall mechanisms [11, 12]. Intact gait control requires the efficient integration of many neural systems and cognitive processes, such as memory, attention and executive function [2, 26, 30]. Therefore, gait dysfunction, particularly during the WWT protocol, could be a potential early indicator of cognitive impairments [31].

Our study included community-residing older adults and may not be generalizable to those who are institutionalized or have cognitive impairments. Older patients with mild cognitive impairment syndrome have impaired performance on dual tasks as well as a higher risk of falls $[32,33]$. Though we lacked a prospective mild cognitive impairment diagnosis procedure, we accounted for this possibility by excluding participants with dementia and significant cognitive impairment as well as adjusting our analyses for baseline Blessed scores. Lack of power analyses has been noted as a limitation of previous dual-task studies [4]. Post hoc power analysis indicated that we had $82 \%$ power to examine WWT and fall associations. While reduced recall of falls is a potential problem [23], we reduced bias by excluding participants with dementia, monitoring falls at short intervals and through high follow-up interview completion rates.

In conclusion, our findings support the predictive validity of WWT tasks for falls in older adults and demonstrate the incremental validity of dual-task over singletask walking assessments. While the WWT individual variables and domains require quantitative methods, they provide valuable insights into improving current risk assessments and developing new therapeutic options. For instance, dual-task performance has been reported to be improved by training even in frail or cognitively impaired older adults $[34,35]$ and could be studied further as a novel falls prevention strategy.

\section{Acknowledgments}

Grant support funding for this project was provided by the National Institute on Aging (AG03949; Principal Investigator: R.B. Lipton).

\section{Disclosure Statement}

There are no conflicts of interest. 


\section{References}

1 Lundin-Olsson L, Nyberg L, Gustafson Y: 'Stops walking when talking' as a predictor of falls in elderly people. Lancet 1997;349:617.

2 Montero-Odasso M, Verghese J, Beauchet O, Hausdorff JM: Gait and cognition: a complementary approach to understanding brain function and the risk of falling. J Am Geriatr Soc 2012;60:2127-2136.

-3 Verghese J, Kuslansky G, Holtzer R, Katz M, Xue XN, Buschke $\mathrm{H}$, et al: Walking while talking: effect of task prioritization in the elderly. Arch Phys Med Rehabil 2007;88:50-53.

-4 Beauchet O, Annweiler C, Dubost V, Allali G, Kressig R, Bridenbaugh S, et al: Stops walking when talking: a predictor of falls in older adults? Eur J Neurol 2009;16:786-795.

-5 Kressig RW, Herrmann FR, Grandjean R, Michel JP, Beauchet O: Gait variability while dual-tasking: fall predictor in older inpatients? Aging Clin Exp Res 2008;20:123-130.

-6 Verghese J, Holtzer R, Lipton RB, Wang C: Quantitative gait markers and incident fall risk in older adults. J Gerontol A Biol Sci Med Sci 2009;64:896-901.

7 Lipton RB, Katz MJ, Kuslansky G, Sliwinski MJ, Stewart WF, Verghese J, et al: Screening for dementia by telephone using the memory impairment screen. J Am Geriatr Soc 2003;51: 1382-1390.

-8 Verghese J, LeValley A, Hall CB, Katz MJ, Ambrose AF, Lipton RB: Epidemiology of gait disorders in community-residing older adults. J Am Geriatr Soc 2006;54:255-261.

-9 Verghese J, Buschke H, Viola L, Katz M, Hall C, Kuslansky G, et al: Validity of divided attention tasks in predicting falls in older individuals: a preliminary study. J Am Geriatr Soc 2002;50:1572-1576.

10 Menz HB, Latt MD, Tiedemann A, Mun San Kwan M, Lord SR: Reliability of the GAITRite ${ }^{\circledR}$ walkway system for the quantification of temporo-spatial parameters of gait in young and older people. Gait Posture 2004;20:20-25.

-11 Lord S, Galna B, Verghese J, Coleman S, Burn $\mathrm{D}$, Rochester L: Independent domains of gait in older adults and associated motor and nonmotor attributes: validation of a factor analysis approach. J Gerontol A Biol Sci Med Sci 2013;68:820-827.

12 Hollman JH, McDade EM, Petersen RC: Normative spatiotemporal gait parameters in older adults. Gait Posture 2011;34:111-118.
13 Verghese J, Wang C, Lipton RB, Holtzer R, Xue X: Quantitative gait dysfunction and risk of cognitive decline and dementia. J Neurol Neurosurgery Psychiatry 2007;78:929-935.

14 Holtzer R, Mahoney JR, Izzetoglu M, Izzetoglu K, Onaral B, Verghese J: fNIRS study of walking and walking while talking in young and old individuals. J Gerontol A Biol Sci Med Sci 2011;66:879-887.

15 Tinetti ME, Baker DI, Mcavay G, Claus EB, Garrett P, Gottschalk M, et al: A multifactorial intervention to reduce the risk of falling among elderly people living in the community. N Engl J Med 1994;331:821-827.

16 Gill TM, Allore HG, Holford TR, Guo Z: Hospitalization, restricted activity, and the development of disability among older persons. JAMA 2004;292:2115-2124.

17 Blessed G, Tomlinson BE, Roth M: The association between quantitative measures of dementia and of senile change in the cerebral grey matter of elderly subjects. Br J Psychiatry 1968;114:797-811.

-18 Yesavage JA, Brink TL, Rose TL, Lum O, Huang V, Adey M, et al: Development and validation of a geriatric depression screening scale: a preliminary report. J Psychiatr Res 1983;17:37-49.

$>19$ Verghese J, Lipton RB, Hall CB, Kuslansky G, Katz MJ, Buschke $\mathrm{H}$ : Abnormality of gait as a predictor of non-Alzheimer's dementia. N Engl J Med 2002;347:1761-1768.

20 Schenkman M, Hughes MA, Samsa G, Studenski S: The relative importance of strength and balance in chair rise by functionally impaired older individuals. J Am Geriatr Soc 1996;44:1441-1446.

-21 Studenski S, Perera S, Wallace D, Chandler JM, Duncan PW, Rooney E, et al: Physical performance measures in the clinical setting. J Am Geriatr Soc 2003;51:314-322.

22 Holtzer R, Friedman R, Lipton RB, Katz M, Xue X, Verghese J: The relationship between specific cognitive functions and falls in aging. Neuropsychology 2007;21:540-548.

23 Ganz DA, Higashi T, Rubenstein LZ: Monitoring falls in cohort studies of communitydwelling older people: effect of the recall interval. J Am Geriatr Soc 2005;53:2190-2194.

24 Verghese J, Ambrose AF, Lipton RB, Wang C: Neurological gait abnormalities and risk of falls in older adults. J Neurol 2010;257:392-398.
25 Yogev囚Seligmann G, Hausdorff JM, Giladi N: The role of executive function and attention in gait. Mov Disord 2008;23:329-342.

26 Holtzer R, Verghese J, Xue X, Lipton RB: Cognitive processes related to gait velocity: results from the Einstein Aging Study. Neuropsychology 2006;20:215-223.

27 Doi T, Makizako H, Shimada H, Yoshida D, Ito $\mathrm{K}$, Kato $\mathrm{T}$, et al: Brain atrophy and trunk stability during dual-task walking among older adults. J Gerontol A Biol Sci Med Sci 2012; 67:790-795.

28 Annweiler C, Beauchet O, Bartha R, Wells JL, Borrie MJ, Hachinski V, et al: Motor cortex and gait in mild cognitive impairment: a magnetic resonance spectroscopy and volumetric imaging study. Brain 2013;136:859-871.

29 Nadkarni NK, Levine B, McIlroy WE, Black SE: Impact of subcortical hyperintensities on dual-tasking in Alzheimer disease and aging. Alzheimer Dis Assoc Disord 2012;26: 28-35.

-30 Herman T, Mirelman A, Giladi N, Schweiger A, Hausdorff JM: Executive control deficits as a prodrome to falls in healthy older adults: a prospective study linking thinking, walking, and falling. J Gerontol A Biol Sci Med Sci 2010;65:1086-1092.

-31 Verghese J, Robbins M, Holtzer R, Zimmerman M, Wang C, Xue X, et al: Gait dysfunction in mild cognitive impairment syndromes. J Am Geriatr Soc 2008;56:12441251.

32 Delbaere K, Kochan NA, Close JC, Menant JC, Sturnieks DL, Brodaty H, et al: Mild cognitive impairment as a predictor of falls in community-dwelling older people. Am J Geriatr Psychiatry 2012;20:845-853.

33 Montero-Odasso M, Muir SW, Speechley M: Dual-task complexity affects gait in people with mild cognitive impairment: the interplay between gait variability, dual tasking, and risk of falls. Arch Phys Med Rehabil 2012;93:293299.

- 34 Andersson AG, Kamwendo K, Seiger A, Appelros P: How to identify potential fallers in a stroke unit: validity indexes of 4 test methods. J Rehabil Med 2006;38:186-191.

- 35 Verghese J, Mahoney J, Ambrose AF, Wang C, Holtzer R: Effect of cognitive remediation on gait in sedentary seniors. J Gerontol A Biol Sci Med Sci 2010;65:1338-1343. 